\title{
The coin operators constructed by QBN Walk and one-dimensional two state quantum walk
}

\author{
Yan Luo, Caishi Wang, Guangbo Yang, Yanyan Wang, Xueqi Nan
}

\begin{abstract}
In this paper, we examine QBN walk and one-dimensional two state quantum walk. We construct coin operators on coin space $H$ by QBN walk and one-dimensional two state quantum walk. We also obtain some formulas about those coin operators.
\end{abstract}

Keywords-QBN walk, one-dimensional two state quantum walk, coin operators.

\section{INTRODUCTION}

The discrete-time quantum walk $(\mathrm{QW})$ as first studied by Ambainis et al.[1], which have found wide application in quantum information, quantum computing, and many other fields [2],[3]. The QW is considered as a quantum generalization of the classical random walk. The random walker in position $\mathrm{x} \in Z=\{0, \pm 1, \pm 2, \ldots\}$ at time $\mathrm{t}(\in\{0,1,2, \ldots\})$ moves to $\mathrm{x}-1$ at time $\mathrm{t}+1$ with probability $\mathrm{p}$, or $\mathrm{x}+1$ with probability $\mathrm{q}(=1-\mathrm{p})$. In the past two decades,quantum walks with a finite number of internal degrees of freedom have been intensively studied and many deep results have been obtained (see [4-6] and references therein). For example, Konno [6] found that a one-dimensional quantum walk with two internal degrees of freedom usually has a limit probability distribution with scaling speed $n$, instead of $\sqrt{n}$, which is far from being Gaussian.

Quantum Bernoulli noises(QBN) are the family of annihilation and creation operators acting on square integrable Bernoulli functionals, which satisfy a canonical anti-commutation relation (CAR) in equal time. In 2016, by using quantum Bernoulli noises, Wang and Ye [7] introduced a discrete-time quantum walk model on the one-dimensional integer lattice $\mathrm{Z}$, which we call the one-dimensional QBN walk below.

In this paper, our work devote to construct coin operators on $H$ by QBN walk and one-dimensional two-state quantum walk and obtain some formulas about those coin operators, which are interesting.

Yan Luo, School of Mathematics and statistics, Northwest Normal University, Lanzhou, Gansu, China.

\section{PRELIMINARIES}

In this section, we briefly recall some notions and results for quantum Bernoulli noises(QBN) walk and one-dimensional two state quantum walk. For details, see $[3,8-11]$ and references therein.

We first introduce the related conclusions of QBN walk.

Let $N$ be the set of all nonnegative integers and $\Gamma$ the finite power set of $N$, namely,

$$
\Gamma=\{\sigma \mid \sigma \subset N \text { and } \# \sigma<\infty\},
$$

where $\# \sigma$ denotes the cardinality of $\sigma$ as a set.

Thoughout, we assume that $(\Omega, F, P)$ is a probability space and $Z=\left(Z_{n}\right)_{n \geq 0}$ is an independent sequence of random variables on $(\Omega, F, P)$, which satisfies that

$$
P\left\{Z=\theta_{n}\right\}=p_{n}, \quad P\left\{Z=-1 / \theta_{n}\right\}=q_{n}, \quad n \geq 0
$$

with $\theta_{n}=\sqrt{q_{n} / p_{n}}, q_{n}=1-p_{n}$ and $0 \leq p_{n} \leq 1$. And, moreover, $F=\sigma\left(Z_{n}, n \geq 0\right)$, the $\sigma$-filed generated by $Z=\left(Z_{n}\right)_{n \geq 0}$. And $Z$ is actually a discrete-time Bernoulli noise.

Let $L^{2}(Z)$ be the space of square integrable complex-valued random variables on $(\Omega, F, P)$.

We denote by $\langle\cdot, \cdot\rangle$ the inner product of $L^{2}(Z)$, and by $\|\cdot$,$\| the corresponding norm. It is known that$ $Z$ has the orthonormal basis

$$
\left\{Z_{\sigma} \mid \sigma \in \Gamma\right\}, \text { where }
$$

$Z_{\varnothing}=1$ and

$$
Z_{\sigma}=\prod_{i \in \sigma} Z_{i}, \sigma \in \Gamma, \sigma \neq \varnothing,
$$

which shows that $L^{2}(Z)$ is an infinite dimensional space.

Lemma 1.[8] For $k \geq 0$, there exists a bounded operator $\partial_{k}$ on $L^{2}(Z)$ such that

$$
\partial_{k} Z_{\sigma}=1_{\sigma}(k) Z_{\sigma \backslash k}, \quad \sigma \in \Gamma,
$$

where $\sigma \backslash k=\sigma \backslash\{k\}$ and $1_{\sigma}(k)$ is the indicator of $\sigma$ a subset of $N$. 


\section{The coin operators constructed by QBN Walk and one-dimensional two state quantum walk}

Lemma 2.[8] For $k \geq 0$, then $\partial_{k}^{*}$, the adjoint operator, has following property:

$$
\partial_{k}^{*} Z_{\sigma}=\left(1-1_{\sigma}(k)\right) Z_{\sigma \cup k,} \quad \sigma \in \Gamma,
$$

where $\sigma \cup k=\sigma \cup\{k\}$.

Lemma 3.[8] Let $k, l \in N$. Then it holds true that

$$
\partial_{k} \partial_{l}=\partial_{l} \partial_{k}, \quad \partial_{k}^{*} \partial_{l}^{*}=\partial_{l}^{*} \partial_{k}^{*}, \quad \partial_{k}^{*} \partial_{l}=\partial_{l} \partial_{k}^{*}(k \neq l)
$$

and

$$
\partial_{k} \partial_{k}=\partial_{k}^{*} \partial_{k}^{*}=0, \quad \partial_{k} \partial_{k}^{*}+\partial_{k}^{*} \partial_{k}=I,
$$

where $I$ is the identity operator on $H$.

For a nonnegative intege $n \geq 0$, we can define, respectively, two self-adjoint operators $L_{n}$ and $R_{n}$ on $H$ in the following manner

$$
L_{n}=\frac{1}{2}\left(\partial_{n}^{*}+\partial_{n}-I\right), \quad R_{n}=\frac{1}{2}\left(\partial_{n}^{*}+\partial_{n}+I\right)
$$

where $I$ is the identity operator on $H$. It then follows from Lemma 3 that the operators $L_{n}, R_{n}, n \geq 0$, form a commutative family, namely

$$
L_{k} L_{l}=L_{l} L_{k}, \quad R_{k} L_{l}=L_{l} R_{k}, \quad R_{k} R_{l}=R_{l} R_{k}, k . l \geq 0
$$

Lemma 4 .[8] For all $n \geq 0, R_{n}+L_{n}$ is a unitary operator on $H$ and moreover it holds that

$$
R_{n}^{2}=R_{n}, \quad R_{n} L_{n}=L_{n} R_{n}=0, \quad L_{n}^{2}=-L_{n}
$$

Now, we indroduce the quantum walk with two coin $e_{1}$ and $e_{2}$ state on the line, which is located at $Z=\{0, \pm 1, \pm 2, \ldots\}$. The quantum system is expressed by a tensor space of two Hilbert space. One is the Hilbert space $l^{2}(Z)$ which describes the position of the quantum walk and it is spanned by the orthogonal normalized basis $\{x: x \in Z\}$, the other is the Hilbert space $C^{2}$ by the orthogonal normalized basis $\left\{e_{1}, e_{2}\right\}$ and it is called coin space. We take the tensor space $l^{2}(Z) \otimes C^{2}$ as the state space of the walk, it is well know that $l^{2}(Z) \otimes C^{2} \cong l^{2}\left(Z, C^{2}\right)$.

\section{MAIN RESULTS}

The QBN walk takes the space $H=L^{2}(Z)$ as its coin space, hence has infinitely many internal degrees of freedom since $H$ is infinite dimensional. And we also know the coin space of one-dimensional two state quantum walk is $C^{2}$.

Definition 1. A pair $(C, D)$ if the sum $C+D$ is unitary and of operators on $C^{2}$ is called a coin op- erator pair $C^{*} D=D^{*} C=0$.

Then a simple calculation gives

$$
C^{*} C+D^{*} D=C C^{*}+C D^{*}=I
$$

We assume that $J: H \otimes C^{2} \rightarrow H$ is a fixed unitary isomorphism. Such a unitary isomorphism exists because $H$ is infinite-dimensional and separable.

For $n \geq 0$, we let

$$
\begin{aligned}
& Q_{(-,-)}^{(n)}=J\left(R_{n} \otimes C\right) J^{-1} \\
& Q_{(-,+)}^{(n)}=J\left(L_{n} \otimes C\right) J^{-1} \\
& Q_{(+,-)}^{(n)}=J\left(R_{n} \otimes D\right) J^{-1} \\
& Q_{(+,+)}^{(n)}=J\left(L_{n} \otimes D\right) J^{-1}
\end{aligned}
$$

From the above definition we can get the following theorem.

Theorem 1. For $n \geq 0$, the four operators defined above is coin operators on $H$. That is, they admit the following operation properties:

(1) $Q_{(-,-)}^{(n)}+Q_{(-,+)}^{(n)}+Q_{(+,-)}^{(n)}+Q_{(+,+)}^{(n)}$ is unitary operator on $H$;

(2) $\left[Q_{(-,-)}^{(n)}\right]^{*} Q_{(-,+)}^{(n)}=0, \quad\left[Q_{(-,-)}^{(n)}\right]^{*} Q_{(+,-)}^{(n)}=0$

$$
\begin{aligned}
& {\left[Q_{(-,-)}^{(n)}\right]^{*} Q_{(+,+)}^{(n)}=0,\left[Q_{(-,+)}^{(n)}\right]^{*} Q_{(+,-)}^{(n)}=0} \\
& {\left[Q_{(-,+)}^{(n)}\right]^{*} Q_{(+,-)}^{(n)}=0, \quad\left[Q_{(+,-)}^{n}\right]^{*} Q_{(+,+)}^{n}=0}
\end{aligned}
$$

Proof. Fist, we prove the $Q_{(-,-)}^{(n)}+Q_{(-,+)}^{(n)}+Q_{(+,-)}^{(n)}$ $+Q_{(+,+)}^{(n)}$ is unitary operator on $H$.

$$
\begin{aligned}
Q_{(-,+)}^{(n)}+Q_{(+,+)}^{(n)} & =J\left(L_{n} \otimes C\right) J^{-1}+J\left(L_{n} \otimes D\right) J^{-1} \\
= & J\left(L_{n} \otimes(C+D)\right) J^{-1}
\end{aligned}
$$

Since $L_{n}$ and $\mathrm{C}+\mathrm{D}$ are unitary operator, thus, $Q_{(-,+)}^{(n)}+Q_{(+,+)}^{(n)}$ is unitary operator. Similary, we have $Q_{(-,-)}^{(n)}+Q_{(-,+)}^{(n)}$ is unitary operator.

Next, we verity property(2). We just need to prove that $\left[Q_{(-,-)}^{(n)}\right]^{*} Q_{(-,+)}^{(n)}=0$. By Lemma 4, we can get

$$
\begin{aligned}
{\left[Q_{(-,-)}^{(n)}\right]^{*} Q_{(-,+)}^{(n)} } & =\left[J\left(R_{n} \otimes C\right) J^{-1}\right]^{*} J\left(L_{n} \otimes C\right) J^{-1} \\
& =J\left(R_{n} \otimes C^{*}\right) J^{-1} J\left(L_{n} \otimes C\right) J^{-1} \\
& =J\left(R_{n} L_{n} \otimes C^{*} C\right) J^{-1} \\
& =0
\end{aligned}
$$

Similary,

$$
\begin{aligned}
& {\left[Q_{(-,-)}^{(n)}\right]^{*} Q_{(+,-)}^{(n)}=\left[Q_{(-,-)}^{(n)}\right]^{*} Q_{(+,+)}^{(n)}=\left[Q_{(-,+)}^{(n)}\right]^{*} Q_{(+,-)}^{(n)}=} \\
& {\left[Q_{(-,+)}^{(n)}\right]^{*} Q_{(+,-)}^{(n)}=\left[Q_{(+,-)}^{(n)}\right]^{*} Q_{(+,+)}^{(n)}=0}
\end{aligned}
$$

Theorem 2. For $n \geq 0$, let $Q_{(-,-)}^{(n)}, Q_{(-,+)}^{(n)}, Q_{(+,-)}^{(n)}$ and 
$Q_{(+,+)}^{(n)}$ be the coin operators on $H$. Then a simple calculation gives

$\left[Q_{(-,)}^{(n)}\right]^{*} Q_{(-,-)}^{(n)}+\left[Q_{(-,+)}^{(n)}\right]^{*} Q_{(-,+)}^{(n)}+\left[Q_{(+,-)}^{(n)}\right]^{*} Q_{(+,-)}^{(n)}+\left[Q_{(+,+)}^{(n)}\right]^{*} Q_{(+,-)}^{(n)}=I$

$Q_{(-,-)}^{(n)}\left[Q_{(-,-)}^{(n)}\right]+Q_{(-,+)}^{(n)}\left[Q_{(-,+)}^{(n)}\right]^{*}+Q_{(+,-)}^{(n)}\left[Q_{(+,-)}^{(n)}\right]^{*}+Q_{(+,-)}^{(n)}\left[Q_{(+,+)}^{(n)}\right]^{*}=I$

Proof. By using (9) and Lemma 4, we have

$\left[Q_{(-,-)}^{(n)}\right]^{*} Q_{(-,-)}^{(n)}+\left[Q_{(-,+)}^{(n)}\right]^{*} Q_{(-,+)}^{(n)}+\left[Q_{(+,-)}^{(n)}\right]^{*} Q_{(+,-)}^{(n)}+\left[Q_{(+,+)}^{(n)}\right]^{*} Q_{(+,-)}^{(n)}$

$=\left[J\left(R_{n} \otimes C\right) J^{-1}\right]^{*} J\left(R_{n} \otimes C\right) J^{-1}$

$+\left[J\left(L_{n} \otimes C\right) J^{-1}\right]^{*} J\left(L_{n} \otimes C\right) J^{-1}$

$+\left[J\left(R_{n} \otimes D\right) J^{-1}\right]^{*} J\left(R_{n} \otimes D\right) J^{-1}$

$+\left[J\left(L_{n} \otimes D\right) J^{-1}\right]^{*} J\left(L_{n} \otimes D\right) J^{-1}$

$=J\left(R_{n}^{2} \otimes C^{*} C\right) J^{-1}+J\left(L_{n}^{2} \otimes C^{*} C\right) J^{-1}$

$+J\left(R_{n}^{2} \otimes D^{*} D\right) J^{-1}+J\left(L_{n}^{2} \otimes D^{*} D\right) J^{-1}$

$=J\left[\left(2 R_{n}-2 L_{n}\right) \otimes\left(C^{*} C+D^{*} D+C^{*} C+D^{*} D\right)\right] J^{-1}$

which together (8) implies (11). Next, we verity formula(12).

$Q_{(-,)}^{(n)}\left[Q_{(-,)}^{(n)}\right]^{*}+Q_{(-,+)}^{(n)}\left[Q_{(-,+)}^{(n)}\right]^{*}+Q_{(+,-)}^{(n)}\left[Q_{(+,-)}^{(n)}\right]^{*}+Q_{(+,-)}^{(n)}\left[Q_{(+,+)}^{(n)}\right]^{*}$

$=J\left(R_{n} \otimes C\right) J^{-1}\left[J\left(R_{n} \otimes C\right) J^{-1}\right]^{*}$

$+J\left(L_{n} \otimes C\right) J^{-1}\left[J\left(L_{n} \otimes C\right) J^{-1}\right]^{*}$

$+J\left(R_{n} \otimes D\right) J^{-1}\left[J\left(R_{n} \otimes D\right) J^{-1}\right]^{*}$

$+J\left(L_{n} \otimes D\right) J^{-1}\left[J\left(L_{n} \otimes D\right) J^{-1}\right]^{*}$

$=J\left(R_{n}^{2} \otimes C C^{*}\right) J^{-1}+J\left(L_{n}^{2} \otimes C C^{*}\right) J^{-1}$

$+J\left(R_{n}^{2} \otimes D D^{*}\right) J^{-1}+J\left(L_{n}^{2} \otimes D D^{*}\right) J^{-1}$

$=J\left[\left(2 R_{n}-2 L_{n}\right) \otimes\left(C C^{*}+D D^{*}+C C^{*}+D D^{*}\right)\right] J^{-1}$

which together (8) implies (12).

\section{CONCLUSIONS REMARK}

As is well know, the Hadamard walk is one-dimensional two-state quantum walk, whose coin space is a two dimensional space $C^{2}$, and we also know the coin space of QBN walk is a infinite dimensional space $H=L^{2}(Z)$. It is interesting that we can construct new coin operators on $H$ by QBN walk and one-dimensional two-state quantum walk, which shares the same coin space with the QBN walk. Then we can examin some interesting properties. The details are considered in elsewhere.

\section{ATCKNOWLEDGEMENT}

This work is supported by National Natural Science Foundation of China (Grant No. 11461061).

\section{REFERENCE}

[1] Ambainis, A., Bach, E., Nayak, A., Vishwanath, A., Watrous, J.:One-dimensional quantum walks[J]. Proceedings of the 33rd Annual ACM Symposium on Theory of Computing, 2001:3749.

[2] J.Kempe. Quantum random walks: An introductory overview[J]. Contemporary Physiscs, 2004, 44(4): 307-327.

[3] N. Konno, P. Biane, L. Bouten, et al. Quantum Walks[J]. Lecture notes in mathematics, 2008.

[4] Venegas-Andraca, S.E. Quantum walks: A comprehensive review[J]. Quantum Inf. Process. 2012, 11, 1015-1106.

[5] Komatsu, T.; Konno, N. Stationary amplitudes of quantum walks on the higher-dimensional integer lattice[J]. Quantum Inf. Process. 2017, 16, 16.

[6] Konno, N. A new type of limit theorems for one-dimensional quantum random walks[J]. J. Math. Soc. Jpn. 2005, 57(4):1179-1195.

[7] Wang, C.S.; Ye, X.J. Quantum walk in terms of quantum Bernoulli noises. Quantum Inf. Process. 2016, 15, 1897-1908.

[8] Wang C, Chai H , Lu Y . Discrete-time quantum Bernoulli noises[J]. Journal of Mathematical Physics, 2010, 51(5):23.

[9] Wang C , Zhang J . Localization of quantum Bernoulli noises[J]. Journal of Mathematical Physics, 2013, 54(10):23-278.

[10] Privault N . Stochastic analysis of Bernoulli processes[J]. Probability Surveys, 2008, 5(1):435483.

[11] Wang C , Lu Y, Chai H . An alternative approach to Privault's discrete-time chaotic calculus[J]. Journal of Mathematical Analysis \& Application -s, 2011, 373(2):643-654.

[12] Machida T . Realization of the probability laws in the quantum central limit theorems by a quantum walk[J]. Quantum information \& computation, 2013, 13(5).

Yan Luo, School of Mathematics and Statistics, Northwest Normal University, Lanzhou, China, Mobile, 86-15693157023.

Caishi Wang, School of Mathematics and Statistics, Northwest Normal University, Lanzhou, China.

Guangbo Yang, School of Mathematics and Statistics, Northwest Normal University, Lanzhou, China.

Yanyan Wang, School of Mathematics and Statistics, Northwest Normal University, Lanzhou, China.

Xueqi Nan, School of Mathematics and Statistics, Northwest Normal University, Lanzhou, China. 